\title{
Time course and mechanisms of left ventricular systolic and diastolic dysfunction in monocrotaline-induced pulmonary hypertension
}

\author{
Jorge Correia-Pinto - Tiago Henriques-Coelho - Roberto Roncon-Albuquerque Jr • \\ André P. Lourenço · Gustavo Melo-Rocha · Francisco Vasques-Nóvoa · \\ Thierry C. Gillebert · Adelino F. Leite-Moreira
}

Received: 23 October 2007/Revised: 27 January 2009/ Accepted: 24 February 2009

(C) Springer-Verlag 2009

\begin{abstract}
Although pulmonary hypertension (PH) selectively overloads the right ventricle (RV), neuroendocrine activation and intrinsic myocardial dysfunction have been described in the left ventricle (LV). In order to establish the timing of LV dysfunction development in $\mathrm{PH}$ and to clarify underlying molecular changes, Wistar rats were studied 4 and 6 weeks after subcutaneous injection of monocrotaline (MCT) $60 \mathrm{mg} / \mathrm{kg}$ (MCT-4, $n=11$; MCT-6, $n=11$ ) or vehicle (Ctrl-4, $n=11$; Ctrl-6, $n=11)$. Acute single beat stepwise increases of systolic pressure were performed from baseline to isovolumetric (LVPiso). This hemodynamic stress was used to detect early changes in LV performance. Neurohumoral activation was evaluated by measuring angiotensin-converting enzyme (ACE) and endothelin-1 (ET-1) LV mRNA levels. Cardiomyocyte apoptosis was evaluated by TUNEL assay. Extracellular
\end{abstract}

J. Correia-Pinto and T. Henriques-Coelho contributed equally to this work.

T. Henriques-Coelho $\cdot$ R. Roncon-Albuquerque Jr •

A. P. Lourenço · F. Vasques-Nóvoa · A. F. Leite-Moreira ( $)$

Department of Physiology, Faculty of Medicine,

University of Porto, Alameda Professor Hernâni Monteiro,

4200-319 Porto, Portugal

e-mail: amoreira@med.up.pt

Present Address:

J. Correia-Pinto · G. Melo-Rocha

School of Health Sciences,

Life and Health Sciences Research Institute (ICVS),

University of Minho, Braga, Portugal

T. C. Gillebert

Department of Cardiology, University of Ghent,

Ghent, Belgium matrix composition was evaluated by tenascin-C mRNA levels and interstitial collagen content. Myosin heavy chain (MHC) composition of the LV was studied by protein quantification. MCT treatment increased RV pressures and $\mathrm{RV} / \mathrm{LV}$ weight ratio, without changing $\mathrm{LV}$ end-diastolic pressures or dimensions. Baseline LV dysfunction were present only in MCT-6 rats. Afterload elevations prolonged $\tau$ and upward-shifted end-diastolic pressure dimension relations in MCT-4 and even more in MCT-6. MHC-isoform switch, ACE upregulation and cardiomyocyte apoptosis were present in both MCT groups. Rats with severe PH develop LV dysfunction associated with ET-1 and tenascin-C overexpression. Diastolic dysfunction, however, could be elicited at earlier stages in response to hemodynamic stress, when only LV molecular changes, such as MHC isoform switch, ACE upregulation, and myocardial apoptosis were present.

Keywords Myosin heavy chain - Endothelin-1 . Angiotensin-converting enzyme $\cdot$ Apoptosis $\cdot$ Tenascin-C . Collagen

\section{Introduction}

Although chronic pulmonary hypertension $(\mathrm{PH})$ selectively overloads the right ventricle (RV), left ventricular (LV) dysfunction also manifests in the course of primary $\mathrm{PH}$ [32], chronic thromboembolism [13] and cor pulmonale [41]. Echocardiography-based studies carried out in $\mathrm{PH}$ patients suggested that one of the mechanisms contributing to LV dysfunction is ventricular interdependence and impaired LV filling [35]. Other studies, however, provide evidences that intrinsic LV myocardial abnormalities also contribute to LV dysfunction in severe PH [11, 12, 24, 33]. 
In fact, the LV of rats with severe longstanding $\mathrm{PH}$ induced by monocrotaline (MCT) presents local autocrine/paracrine system activation and muscle strips dissected from the LV myocardium show, in vitro, negative force-frequency relationships (FFR) [33]. Intrinsic LV myocardial abnormalities might help to explain late recovery of LV filling after single-lung transplantation in patients with $\mathrm{PH}$, even if LV geometry and RV function are immediately restored [50]. They might explain as well, why combined heartlung transplantation is favored for $\mathrm{PH}$ patients when severe impairment of LV function is present, since LV function may not sufficiently recover after transplantation of the lungs alone [39].

The timing of development and the underlying mechanisms of intrinsic LV myocardial dysfunction were incompletely investigated so far. In the present study, we analyzed biventricular hemodynamics, LV myosin heavy chain (MHC) protein isoforms, LV myocardial expression of genes involved in neurohumoral activation (angiotensin-converting enzyme and endothelin-1), extracellular matrix remodelling (tenascin- $\mathrm{C}$ gene expression and interstitial fibrosis) and apoptosis (TUNEL assay), 4 and 6 weeks after MCT injection. Hemodynamics were studied at baseline and in response to single-beat afterload elevations, which allow the detection of diastolic dysfunction that may not be evident during evaluation at rest, but is revealed during exercise or hemodynamic stress [9, $10,19,20,26,48]$.

\section{Methods}

\section{Animal protocol}

The investigation conforms to the Guide for the Care and Use of Laboratory Animals published by the US National Institutes of Health (NIH Publication No. 85-23, revised 1996) and the experiments were performed according to the Portuguese law on animal welfare. Seven-weeks-old male Wistar rats ( $n=58$; Charles-River, Barcelona, Spain) were housed in groups of three per cage in a controlled environment under a 12:12 h light/dark cycle at a room temperature of $22^{\circ} \mathrm{C}$. Rats randomly received, a subcutaneous injection of monocrotaline (MCT, $60 \mathrm{mg} / \mathrm{kg}$; Sigma Chemical, St. Louis, MO, USA; $n=36$ ) or an equal volume of vehicle ( $\mathrm{Ctrl}, n=22 ; 1 \mathrm{~mL} / \mathrm{kg}$ ). Animals had free supply of food and water. Hemodynamic studies and collection of samples for molecular and histological studies were carried out, for control and MCT-treated rats, at days 22,24 or 26 (fourth week), Ctrl-4 $(n=11)$ and MCT-4 ( $n=11$ ), respectively, and at days 36,38 or 40 (sixth week) after injection, Ctrl-6 $(n=11)$ and MCT-6 $(n=11)$, respectively.
Hemodynamic studies

\section{Experimental preparation}

Animals were anesthetized with an intraperitoneal injection of $60 \mathrm{mg} / \mathrm{kg}$ pentobarbital sodium and additional boluses of $20 \mathrm{mg} / \mathrm{kg}$ when needed, placed over a heating pad, and mechanically ventilated (Harvard Rodent Ventilator model 683) through a tracheostomy. The right jugular vein was cannulated and a pre-warmed $0.9 \% \mathrm{NaCl}$ solution was administrated to compensate for perioperative fluid losses. The heart was exposed through a median sternotomy and the pericardium widely opened. The ascending aorta was dissected and surrounded by a silk thread in order to transiently occlude it during the experimental protocol. RV pressure was measured with a $2 \mathrm{~F}$ high-fidelity micromanometer (SPR-324, Millar Instruments, Houston, TX, USA) inserted through the RV free wall into the RV cavity and LV pressure with a $3 \mathrm{~F}$ high-fidelity micromanometer (SPR-407, Millar Instruments, Houston, TX, USA) inserted through an apical puncture into the LV cavity. LV septal to free wall diameter was recorded with ultrasonic crystals using a sonomicrometer amplifier (Triton Technology, San Diego, CA, USA). One crystal was placed into the left border of the interventricular septum and the other on the epicardial surface of the LV free wall, as previously described [3, 4]. A limb ECG (II) was recorded throughout. After the instrumentation period (typically $60 \mathrm{~min}$ ), the animal was allowed to stabilize for $15 \mathrm{~min}$ before the beginning of the experimental protocol. Recordings were made with ventilation suspended at end-expiration. Parameters were converted on line to digital data with a sampling frequency of $1,000 \mathrm{~Hz}$.

\section{Data analysis}

Peak rates of $L V$ and $\mathrm{RV}$ pressure rise $\left(\mathrm{d} P / \mathrm{d} t_{\max }\right)$ and fall $(\mathrm{d} P /$ $\left.\mathrm{d} t_{\min }\right)$ were determined. $\mathrm{RV}$ and $\mathrm{LV}$ pressures were measured at end-diastole (EDRVP and EDLVP, respectively) and at peak-systole $\left(\mathrm{RVP}_{\max }\right.$ and $\left.\mathrm{LVP}_{\max }\right)$. Relaxation rate was estimated with the time constant $\tau$, by fitting isovolumetric $\mathrm{LV}$ pressure fall to a monoexponential function. From baseline to isovolumetric, multiple graded LV pressure elevations were randomly performed by abruptly clamping the ascending aortic root starting in the diastole separating two heartbeats, as previously described $[3,4,25,31]$. Beats preceding aortic obstruction were designated as baseline heartbeats and those immediately following it as test beats. Along with the baseline hemodynamics, four afterloaded testbeats with a peak systolic pressure of approximately 60,70 , 80 , and 90 of the isovolumetric pressures, and the isovolumetric test-beat were selected for analysis. LV septum to free wall diameter was measured at end-diastole. Afterload- 
induced shifts of the end-diastolic pressure-dimension relation (EDPDR) were assessed by measuring diastolic pressures at matched dimensions, close to end-diastole, as previously described $[3,4,29,30]$.

At the end of the hemodynamic study animals were euthanized with anesthetic overdose, the position of crystals and manometers was verified, the RV was carefully dissected from the interventricular septum and LV free wall, and transmural samples of LV free wall (excluding the septum) were collected for molecular studies. Two MCT-6 rats died during hemodynamic instrumentation, having been excluded from analysis.

Morphometric determination of cardiac fibrosis

Transverse-sections of paraffin-embedded, formalin-fixed specimens encompassing the LV free-wall were stained with Masson's Trichrome and photographed with a digital camera (Leica DFC320) in additional animals $(n=5$ per group). A 330-point grid was superimposed on ten fields $(400 \times)$ randomly selected on each section. Two independent blinded observers rated the images and the area-percent of blue staining, indicative of fibrosis, was calculated as follows: (total number of blue-positive points/total number of points) $\times 100$.

\section{Detection of apoptotic cardiomyocytes}

To assess the extent of apoptosis the terminal deoxynucleotidyl-transferase-mediated dUTP nick end-labeling (TUNEL) assay was used (CardioTACS ${ }^{\mathrm{TM}}$ in situ Apoptosis Detection Kit, R\&D Systems, Minneapolis, MN, USA). Briefly, after deparaffinization tissue slides were immersed in phosphate-buffered saline (PBS) pH 7.4 and then permeabilized with proteinase $\mathrm{K}$ for $20 \mathrm{~min}$ at room temperature. Endogenous peroxidase activity was quenched using 5.0\% hydrogen peroxide. Then, specimens were incubated in TdT labeling buffer for $5 \mathrm{~min}$. After that, slides were incubated with a mix containing the terminal deoxynucleotidyl transferase (TdT), $\mathrm{Mn}^{2+}$ and biotinylated nucleotides for $1 \mathrm{~h}$ at $37^{\circ} \mathrm{C}$, blocked with stop buffer, and incubated with streptavidin-HRP for $10 \mathrm{~min}$ at room temperature. After washing in PBS, the slides were finally developed using TACS Blue Label. Nuclear staining by Nucler Fast Red was performed as counterstaining. Positive control of the TUNEL assay was generated by staining by the treatment of the samples with TACS-Nuclease ${ }^{\mathrm{TM}}$ prior to the labeling protocol. Negative control of the TUNEL assay was confirmed by staining of the heart tissue in the same manner without TdT. We used three sections obtained at a distance of $100 \mu \mathrm{m}$ from each tissue block. TUNEL-positive cardiomyocytes were counted in at least 50 optical fields $(400 \times)$ of each specimen. The apoptotic rate was expressed as a percentage of apoptotic cells of all cardiomyocytes per field.

Molecular studies

\section{Relative quantification of $m R N A$}

Two-step Real-time RT-PCR was performed as previously described [14, 15, 33, 40]. Briefly, after total mRNA extraction (no. 74124, QIAGEN), standard curves were obtained using graded dilutions of a rat cardiac tissue sample. The starting mRNA quantities were correlated $(R \geq 0.98)$ with the respective threshold cycles, for each gene. Threshold cycles were automatically calculated by software (LightCycler, ROCHE) using the second derivative maximum method. An equal amount of mRNA from every sample underwent two separate two-step real time RT-PCR experiments for each gene, using SYBR green as marker (no. 204143, QIAGEN). Glyceraldehyde-3-phosphate dehydrogenase (GAPDH) was used as internal control since its mRNA levels were similar in the studied groups. Results are normalized for GAPDH and presented in arbitrary units. The arbitrary unit was set as the mean obtained for the Ctrl-4 group. Specific PCR primer pairs for the studied genes were: endothelin-1 (ET-1), fw 5'-CCA TGC AGA AAG GCG TAA AAG- ${ }^{\prime}$, rev $5^{\prime}$-CGG GGC TCT GTA GTC AAT GTG-3'; angiotensin-converting enzyme (ACE), fw $5^{\prime}$-GCA GGC CAG CAG GGT CCA CTA CAC- $3^{\prime}$, rev $5^{\prime}$-GAC CTC GCC ATT CCG CTG ATT CT-3'; tenascin-C, fw 5'-TCT CCG GTG TAG CCC TCG TCA-3' ${ }^{\prime}$, rev $5^{\prime}$-CAC CAT GGC CGC TGT CTC AA$3^{\prime}$; and GADPH, fw $5^{\prime}$-TGG CCT TCC GTG TTC CTA CCC-3', rev $5^{\prime}$-CCG CCT GCT TCA CCA CCT TCT- $3^{\prime}$.

\section{Relative quantification of myosin heavy-chain isoforms}

Total protein $(15 \mu \mathrm{g})$ was separated by SDS-PAGE $(3.0 \%$ stacking and 5.0\% running polyacrylamide gels). Electrophoresis was carried out at constant voltage $(60 \mathrm{~V})$ for $\sim 270$ min (no. 165-3301, BIO-RAD), allowing the separation of myosin heavy-chain isoforms $\alpha$ and $\beta$ isoforms in two distinct bands visible at $\sim 200 \mathrm{KDa}$. Gels were silverstained following the manufacturer's instructions (no. 1610449, BIO-RAD) and the relative amount of the myosin heavy-chain isoforms in each sample was quantified by densitometry (Multilmage Light Cabinet; ALPHA INNOTECH CORPORATION).

Statistical analysis

Values were expressed as mean \pm SEM. Differences in baseline hemodynamic parameters, morphology, and molecular studies were evaluated by two-way ANOVA. 
Three-way ANOVA was employed to compare data from graded afterload elevations. Holm-Sidak's method was used for multiple comparisons when significant differences were detected. Statistical significance was assumed at a two-tailed value of $P<0.05$.

\section{Results}

All MCT-6 animals presented clinical signs of congestive heart failure such as lethargic behavior, ruffled fur, cachexia, tachypnea and severe breathing effort, pleural effusion, and ascites. MCT-4 animals behaved similarly to control animals. Mortality was null in Ctrl-4 and Ctrl-6, $33 \%$ in MCT-4 and 70\% in MCT-6. No significant differences were observed between Ctrl- 4 and Ctrl- 6 for morphometric, hemodynamic and molecular parameters.

Baseline hemodynamics and morphometry

MCT treatment resulted in progressively increasing RV peak-systolic pressures (Fig. 1a). Increased RV pressures were accompanied by selective RV hypertrophy, as denoted by the progressive increase of the RV to LV plus interventricular septum weight ratio (Fig. 1b). RV end-diastolic pressures were significantly increased in MCT-6 (4.9 \pm 1.0 vs. $1.9 \pm 0.5,0.5 \pm 0.3$, and $1.1 \pm 0.6 \mathrm{mmHg}$, in MCT-4, Ctrl-4, and Ctrl-6, respectively). Compared with the other groups, MCT-6 also presented lower body weight and heart rate $(216 \pm 9$ vs. $287 \pm 7,265 \pm 20$, and $270 \pm$ 20 beats/min, in MCT-4, Ctrl-4, and Ctrl-6, respectively).

Both systolic (Fig. 2) and diastolic parameters (Fig. 3) were preserved at baseline in the LV of MCT-4. MCT-6 animals, however, presented low peak LV systolic pressure and disturbed indexes of both LV contractility (Fig. 2), such as reduced peak isovolumetric pressures and $\mathrm{d} P / \mathrm{d} t_{\max }$, and LV relaxation (Fig. 3), such as reduced $\mathrm{d} P / \mathrm{d} t_{\min }$ and prolonged time constant $\tau$. Despite these hemodynamic disturbances neither the EDLVP nor the EDLVD were changed (Fig. 3).

Response of diastolic function to afterload elevations

Both MCT-4 and MCT- 6 revealed an impaired response of diastolic function to afterload elevations (Fig. 4). This figure displays changes in the time constant $\tau$ (panel A) and shifts of the EDPDR (panel B) with increasing afterloads. Effects on $\tau$ are expressed by the ratio of test to baseline beats values. Time constant $\tau$ was differently affected by afterload in control and in MCT-treated rats. Control animals responded to afterload elevations with shortening of $\tau$ (acceleration of pressure fall) for almost the entire range of systolic LV pressures, apart from isovolumetric beats that showed a non-significant change in $\tau$. On the contrary,
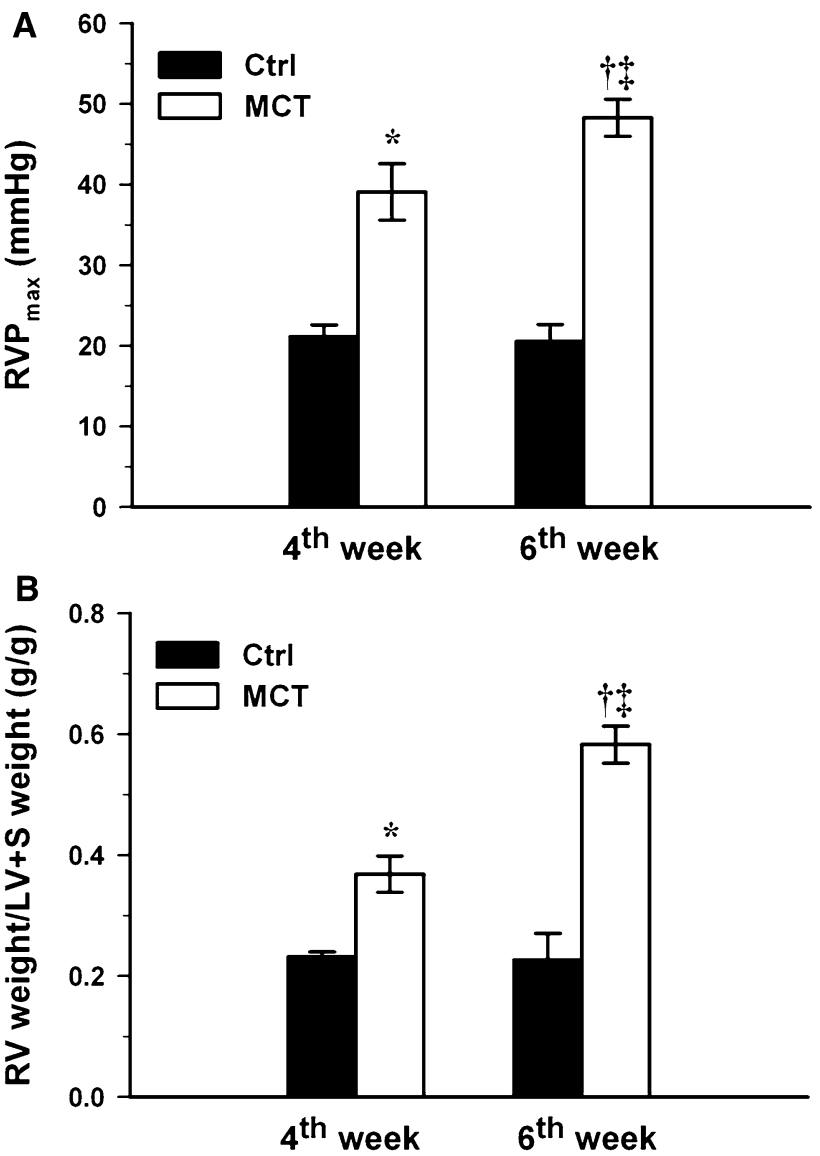

Fig. 1 Development of pulmonary hypertension and selective RV hypertrophy in response to monocrotaline (MCT) injection. a Peak systolic right ventricular pressures $\left(\mathrm{RVP}_{\max }\right)$. b Right ventricular (RV) weight/left ventricular plus interventricular septum $(\mathrm{LV}+\mathrm{S})$ weight ratio. $\mathrm{RVP}_{\max }$ progressively increased in MCT-4 and MCT-6; RV weight to LV weight ratio also increased in MCT-4 and MCT-6. $P<0.05$ : * vs. Ctrl-4; $\dagger$ vs. Ctrl- 6 ; $\$$ vs. MCT-4 ( $n=6$ in each group)

MCT-treated animals responded to afterload elevations with a prolongation of $\tau$ (slowing of pressure fall). This slowing was observed only with the two highest afterload levels in MCT-4, while in MCT-6 such slowing was more pronounced and present over the entire range of systolic LV pressures. With regard to the EDPDR, in response to afterload elevations, it remained unaffected in control animals, but was upward shifted in both MCT-4 and MCT6 groups. This upward shift was of similar magnitude in MCT-4 and MCT-6 (Fig. 4b), but occurred at much lower systolic LV pressures in the later. Representative pressuredimension loops are presented (Fig. 5).

Neurohumoral activation

The expression of genes involved in autocrine/paracrine systems was upregulated in the LV myocardium of MCTtreated rats compared with controls (Fig. 6). Angiotensinconverting enzyme mRNA levels were progressively 

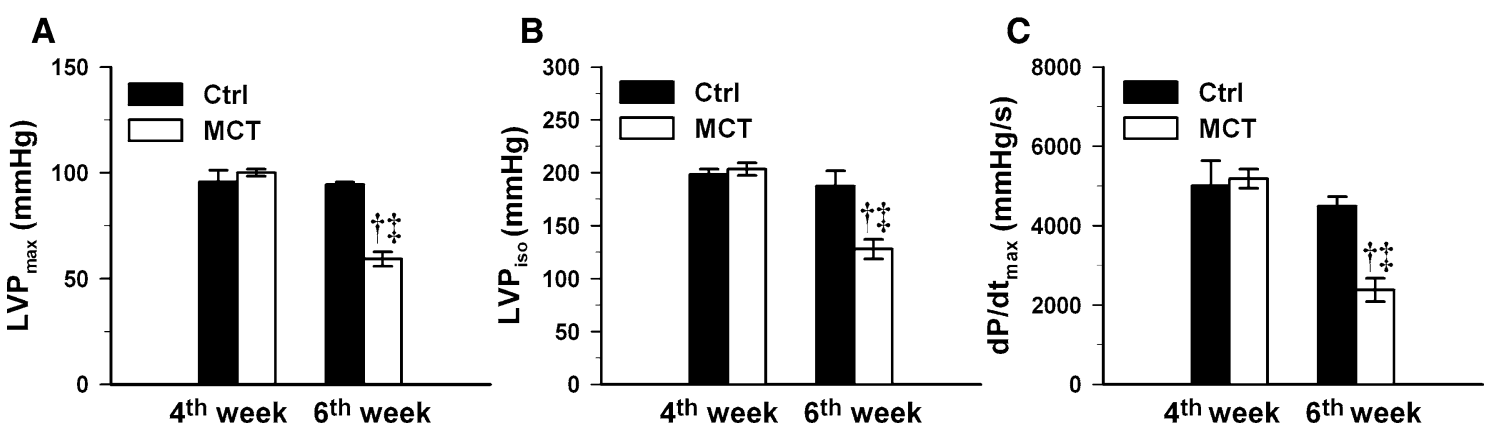

Fig. 2 Left ventricular (LV) systolic hemodynamic parameters. a Peak systolic LV pressure $\left(\mathrm{LVP}_{\max }\right)$. b Peak isovolumetric LV pressure $\left(\mathrm{LVP}_{\text {iso }}\right)$. c Peak rate of $\mathrm{LV}$ pressure rise $\left(\mathrm{d} P / \mathrm{d} t_{\max }\right) . P<0.05: *$ vs. Ctrl- 4 ; $\dagger$ vs. Ctrl-6; $\ddagger$ vs. MCT-4 $(n=6$ in each group)

Fig. 3 Left ventricular (LV) diastolic hemodynamic parameters. a End diastolic left ventricular (LV) pressure (EDLVP). b End diastolic LV dimensions (EDLVD). c Peak rate of LV pressure fall $(\mathrm{d} P /$ $\left.\mathrm{d} t_{\text {min }}\right)$. d LV time constant $\tau$. Significant differences were found for $\mathrm{d} P / \mathrm{d} t_{\min }$ and $\tau$, whereas no differences were observed for EDLVP and EDLVD. $P<0.05: *$ vs. Ctrl4 ; $\dagger$ vs. Ctrl- 6 ; $\$$ vs. MCT-4 ( $n=6$ in each group)
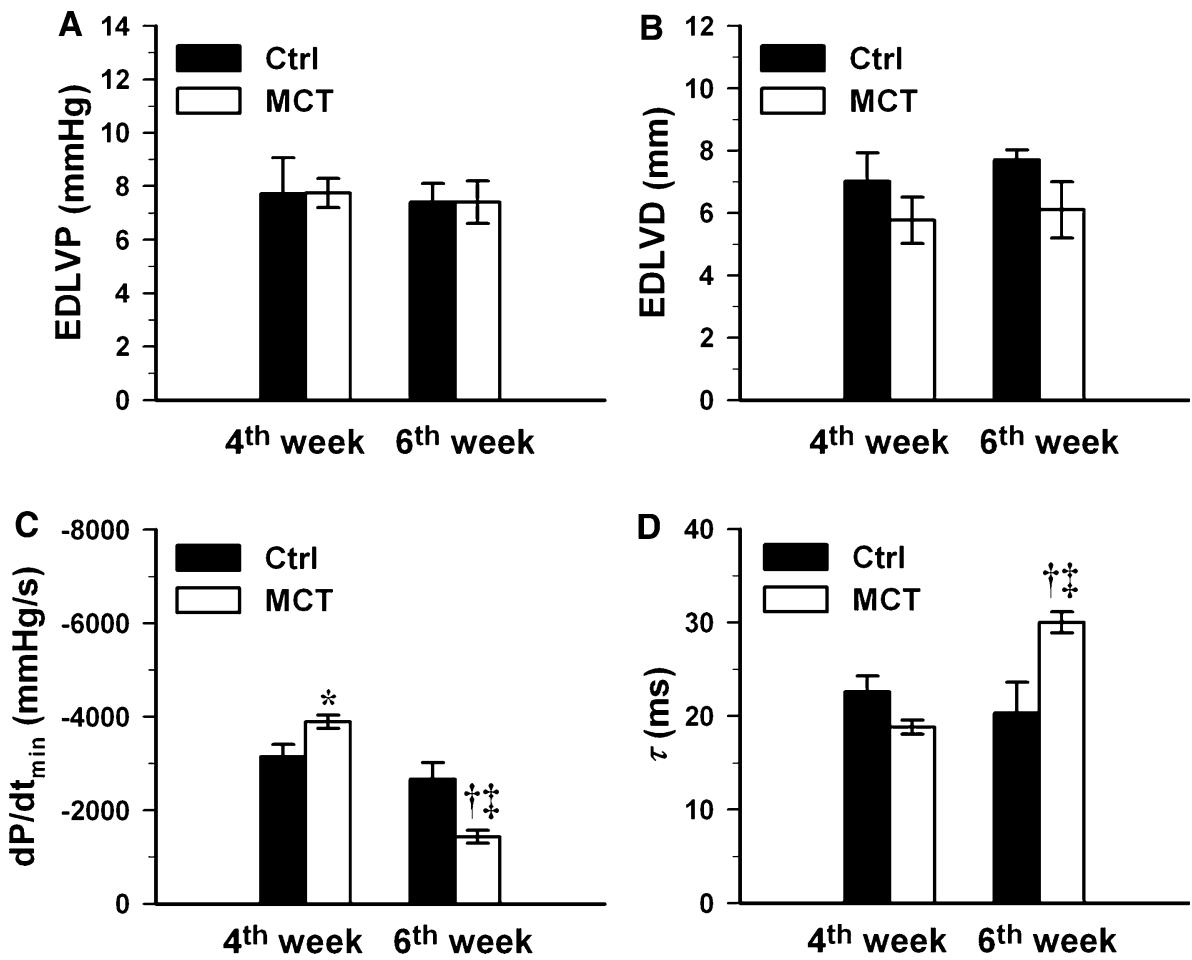

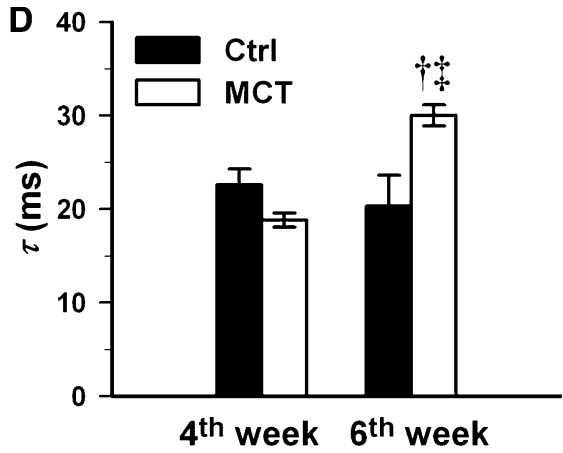

increased in MCT-4 and MCT-6, whereas endothelin-1 expression was only elevated in MCT-6.

\section{Apoptosis}

Apoptosis was significantly augmented in LV myocardium of both MCT-4 and MCT-6 groups, as compared with controls (Fig. 7a). In the representative examples presented in Fig. 7b apoptotic cardiomyocytes can be easily identified in MCT groups.

\section{Extracellular matrix composition}

Regarding the extracellular matrix composition, we evaluated the gene expression of tenascin- $C$, an extracellular matrix glycoprotein as well as the LV interstitial collagen content. Both were significantly increased only in MCT-6 group (Fig. 8).

LV myofilaments

With regard to myofilament composition, a significant elevation of the relative expression of myosin heavy-chain $\beta$-isoform was observed in both MCT-4 and MCT-6 LV myocardium compared with Ctrl-4 and Ctrl-6, respectively (Fig. 9).

\section{Discussion}

The present study was undertaken to investigate the onset and progression of $\mathrm{LV}$ dysfunction in the course of $\mathrm{PH}$ and 

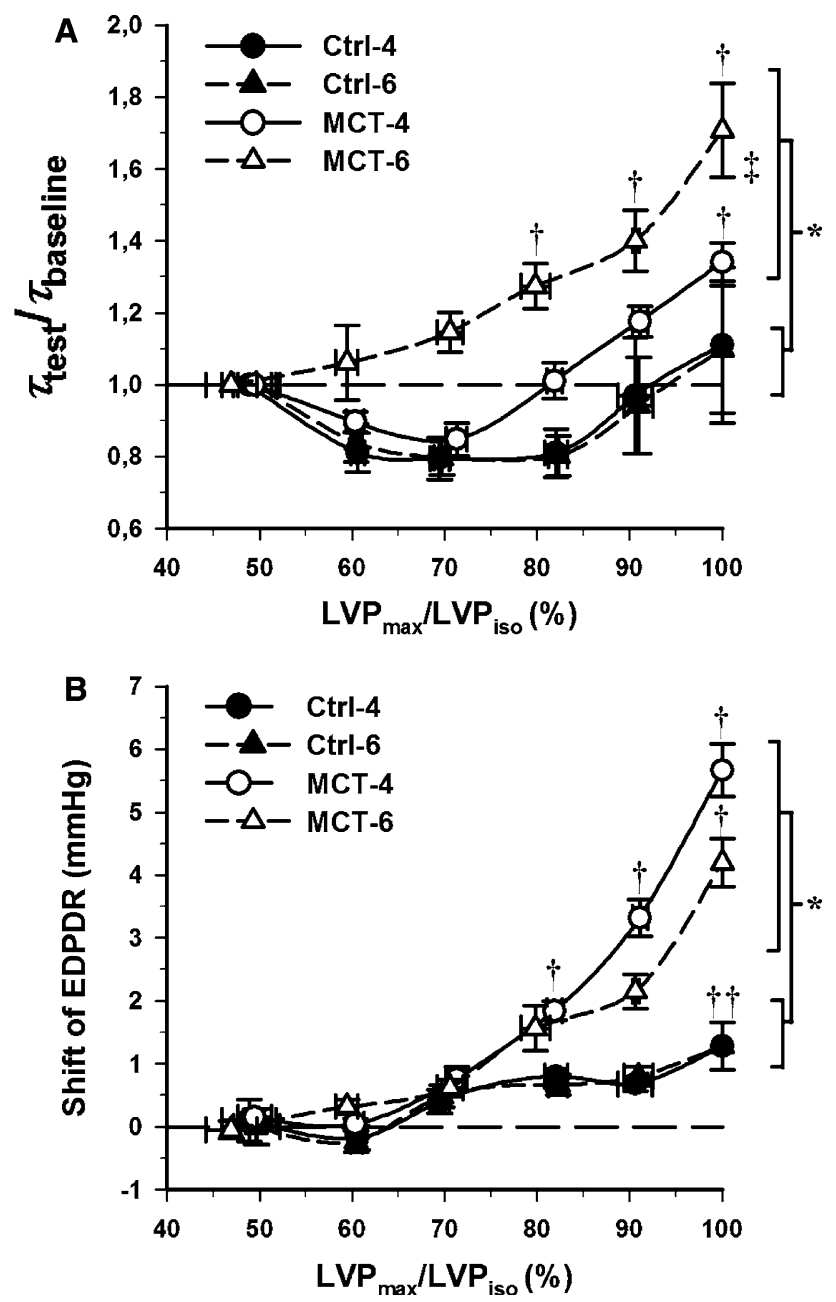

Fig. 4 Effects of acute left ventricular (LV) afterload elevations on LV diastolic function. a Fractional changes in time constant $\tau\left(\tau_{\text {test }} /\right.$ $\left.\tau_{\text {baseline }}\right)$. b Upward shift in the end-diastolic pressure-dimension relation (EDPDR). Six beats with progressively higher peak systolic $\mathrm{LV}$ pressure $\left(\mathrm{LVP}_{\max }\right)$ are represented: baseline, test-beats with a peak systolic LV pressure corresponding to $60,70,80$, and $90 \%$ of the isovolumetric beat, and the isovolumetric test-beat $\left(\mathrm{LVP}_{\text {iso }}\right) ; n=6$ in each group. MCT-treated rats, distinctly from controls $(* P=0.002)$, presented a prolongation of the time constant $\tau$ in test beats compared with baseline beat $(\dagger P<0.01)$, while controls presented an overall acceleration of relaxation and shortening of $\tau$, with the exception of the isovolumetric test beats. In MCT- 6 the changes in $\tau$ were more striking and observed for lower afterload levels than in MCT-4 $(\ddagger P=0.007)$. The upward shift of EDPDR in test-beats compared with baseline beat $(\uparrow P<0.01)$ was more pronounced and observed for lower afterload levels in MCT-treated rats compared with controls $(* P=0.008)$

the underlying myocardial mechanisms, namely neurohumoral activation, extracellular matrix composition and apoptosis. A hemodynamic stress test with single-beat afterload elevations was used to detect early, load dependent, changes in LV diastolic function in animals with MCT induced PH.

MCT-treated rats progressively developed $\mathrm{PH}$, as evaluated by $\mathrm{RVP}_{\max }$, selective RV myocardial hypertrophy, and increased EDRVP. As previously reported [33], MCT6 rats showed clinical signs of congestive heart failure, disturbed indexes of LV contractility and relaxation, and increased expression of ACE and ET-1 in the LV myocardium. A more detailed discussion on the possible causes of neuroendocrine activation in the non-overloaded LV is provided elsewhere [33]. MCT-6 animals also presented reductions in body weight and $\mathrm{LVP}_{\max }$, previously interpreted as consequence of continuous severe sickness [36], as well as, diminished heart rate, suggesting an attenuation of the sympathetic nervous system response [25, 33]. In contrast, MCT-4 rats showed no baseline LV hemodynamic changes. Nonetheless, single afterloaded test-beats revealed diastolic function impairment in MCT-4. Systolic performance, as assessed by the contractility indexes $\mathrm{LVP}_{\text {iso }}$ and $\mathrm{d} P / \mathrm{d} t_{\max }$, was unchanged, but diastolic dysfunction was elicited in response to increased afterload (afterload-induced diastolic dysfunction; [29, 30]). Diastole was evaluated with the time constant of isovolumetric relaxation $\tau$ and the end-diastolic pressure-diameter relation (EDPDR), the former evaluates active relaxation while the latter is an in vivo estimate of myocardial stiffness. The dynamic process of myocardial relaxation goes on from the ejection phase to the early filling period. The main hemodynamic manifestation of myocardial relaxation, however, is LV pressure fall and its analysis, through the time constant $\tau$, allows a description of the course of myocardial relaxation [26]. Changes in afterload modify $\tau$ and the rate of LV pressure fall. While in healthy hearts small afterload elevations accelerate LV pressure fall and only marked afterload increases slow it, in failing hearts even slight increases in afterload may induce slower relaxation. At a similar systolic LV pressure, in failing hearts the relative load, defined as the ratio of systolic LV pressure to isovolumetric LV pressure, is higher, the afterload reserve is surpassed and afterload mismatch yields a pronounced slowing of LV pressure fall, compared with healthy hearts $[10,31]$.

Diastolic dysfunction is commonly defined, in hemodynamic terms, by an upward displacement of the EDPDR [26], which reflects a decrease in diastolic distensibility [13]. Classically, it was held that only chronic conditions that altered the compliance and distensibility of the LV could change the filling conditions to the point of modifying the EDPDR. However, more recent work demonstrated that the EDPDR could be acutely shifted by load [25, 31] and neurohumoral stimulation [27, 28, 37]. Afterload mismatch induces incomplete myocardial relaxation that can be large enough to increase LV pressure at end-diastole and cause diastolic dysfunction. A slower course of myocardial relaxation and the decrease in time available for the ventricle to relax are the major underlying mechanisms of afterload-induced diastolic dysfunction [29, 


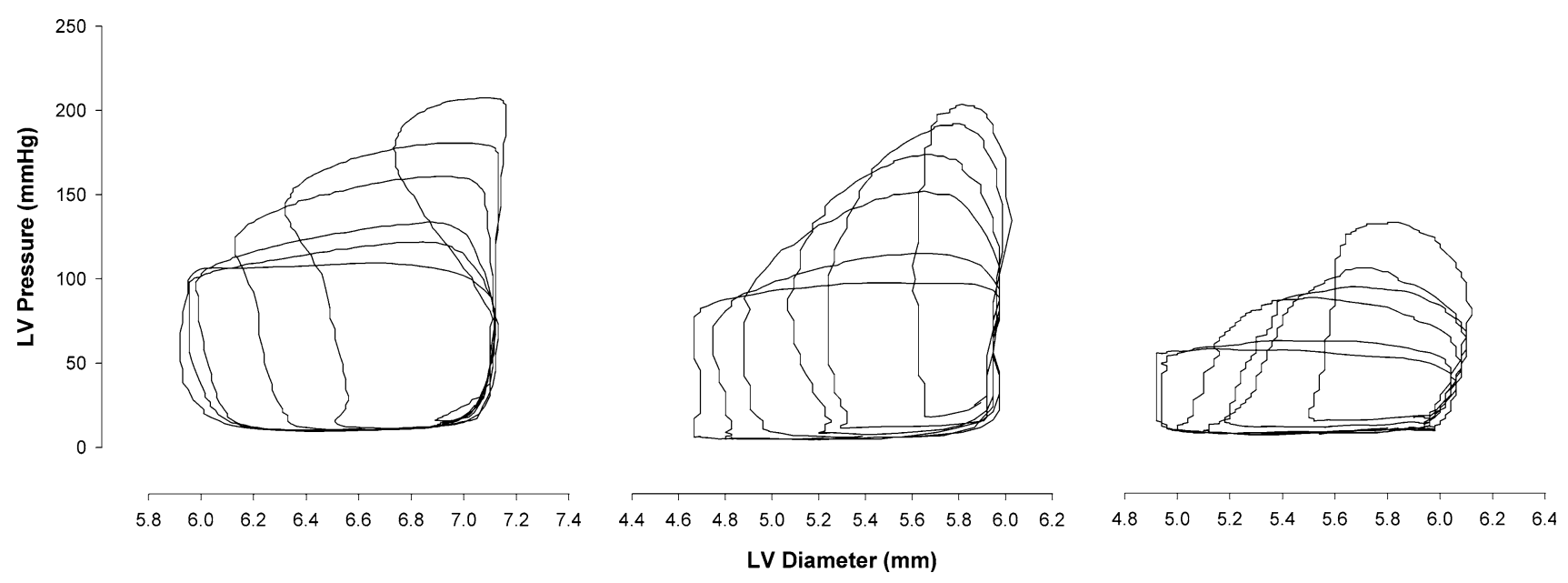

Fig. 5 Representative examples of left ventricular pressure-dimension (LVPD) loops of a Ctrl-4 (left), a MCT-4 (centre) and a MCT-6 (right) animal at increasing afterload levels, from baseline up to isovolumetric. Peak systolic pressures are similar in Ctrl-4 and MCT-4 animals, but are clearly decreased in the MCT-6 animal. On the other side, diastolic pressures are nicely matched at all afterload levels in the control animal, but become progressively higher as afterload increases, both in MCT-4 and MCT-6 animals. As Ctrl-4 and Ctrl-6 animals were entirely similar only a representative example of Ctrl-4 is given

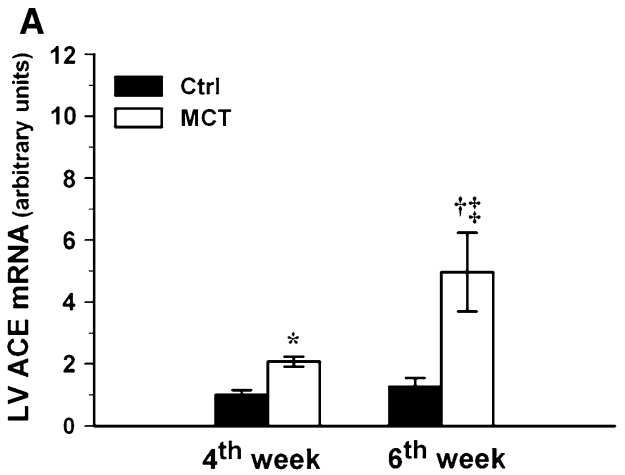

Fig. 6 Left ventricular (LV) mRNA levels of angiotensin-converting enzyme (ACE; a) and endothelin-1 (ET-1; b) in rats with monocrotaline (MTC)-induced pulmonary hypertension (MCT-4, $n=6$; MCT-6, $n=6$ ) and controls (Ctrl-4, $n=6$; Ctrl-6, $n=6$ ), 4 and 6 weeks after injection, respectively. Results are expressed as

30]. Compared with control rats, MCT-treated rats developed prolongation of time constant $\tau$ and a larger upward shift of the EDPDR indicating that afterload mismatch occurred at lower relative loads. Curiously, although MCT6 , unlike MCT-4, presented prolongation of $\tau$ at baseline that was aggravated with every step up in relative load, there were no differences between the two groups regarding the upward shift of the EDPDR induced by afterload. One possible reason is the difference in heart rates. Inappropriate increases in heart rate are known to upwardly displace the EDPDR even if relaxation rate is normal [49]. Conversely, the reduction in the heart rate of MCT-6 may have partly reverted afterload-dependent diastolic dysfunction by increasing the time available for relaxation and the extent of relaxation. Animals and patients with $\mathrm{PH}$ are known to respond badly to hemodynamic stress $[19,23$,

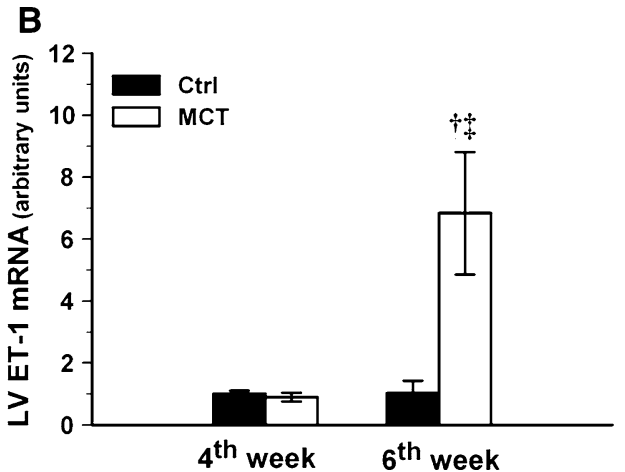

arbitrary units after normalization for GAPDH. The arbitrary unit was set as the average value of the Ctrl-4 group. ACE mRNA levels progressively increased in MCT- 4 and MCT- 6 , whereas ET- 1 mRNA levels were only increased 6 weeks after MCT injection $P<0.05$ : * vs. Ctrl- $4 ; \dagger$ vs. Ctrl-6; $\$$ vs. MCT-4

45]. The present results suggest that increased systemic blood pressures may induce, or contribute to, hemodynamic deterioration in $\mathrm{PH}$ by leading to, or worsening of, LV diastolic dysfunction.

One of the most likely subcellular candidates for myocardial dysfunction is the altered myofilament composition. MCT-treated rats presented myosin heavy-chain isoform switch in the LV myocardium. The slower $\beta$-isoform was increased in the LV of both MCT-4 and MCT-6, as previously reported in different models of RV hypertrophy $[17,43,46,47]$. Changes in the expression of enzymes involved in bioenergetics and cell metabolism such as pyruvate dehydrogenase kinase- 4 , acetyl-CoA dehydrogenase and mitochondrial ATP synthase $[42,43]$ as well as a reduced capillary proliferation [21] have also been described in the LV of PH rats. Contrastingly, calcium kinetics 
Fig. 7 Left ventricular (LV) apoptosis is expressed as TUNEL-positive cell percentage (a). There is a significant increase in apoptosis in both MCT-4 and MCT-6 groups $(P<0.05: *$ vs. Ctrl-4; $\uparrow$ vs. Ctrl-6). Representative examples from rats with monocrotaline-induced pulmonary hypertension (MCT) and controls (Ctrl) 4 and 6 weeks after injection are presented in b (arrows apoptotic cells)

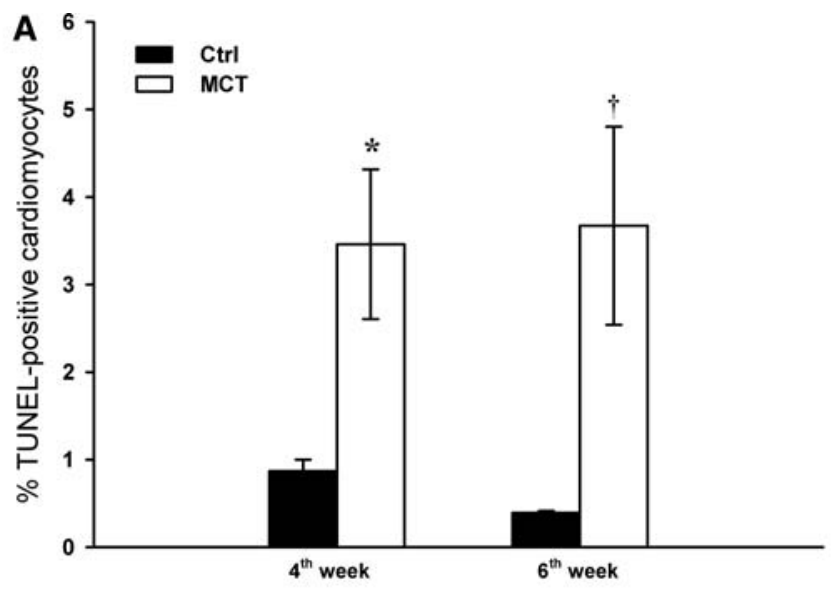

B
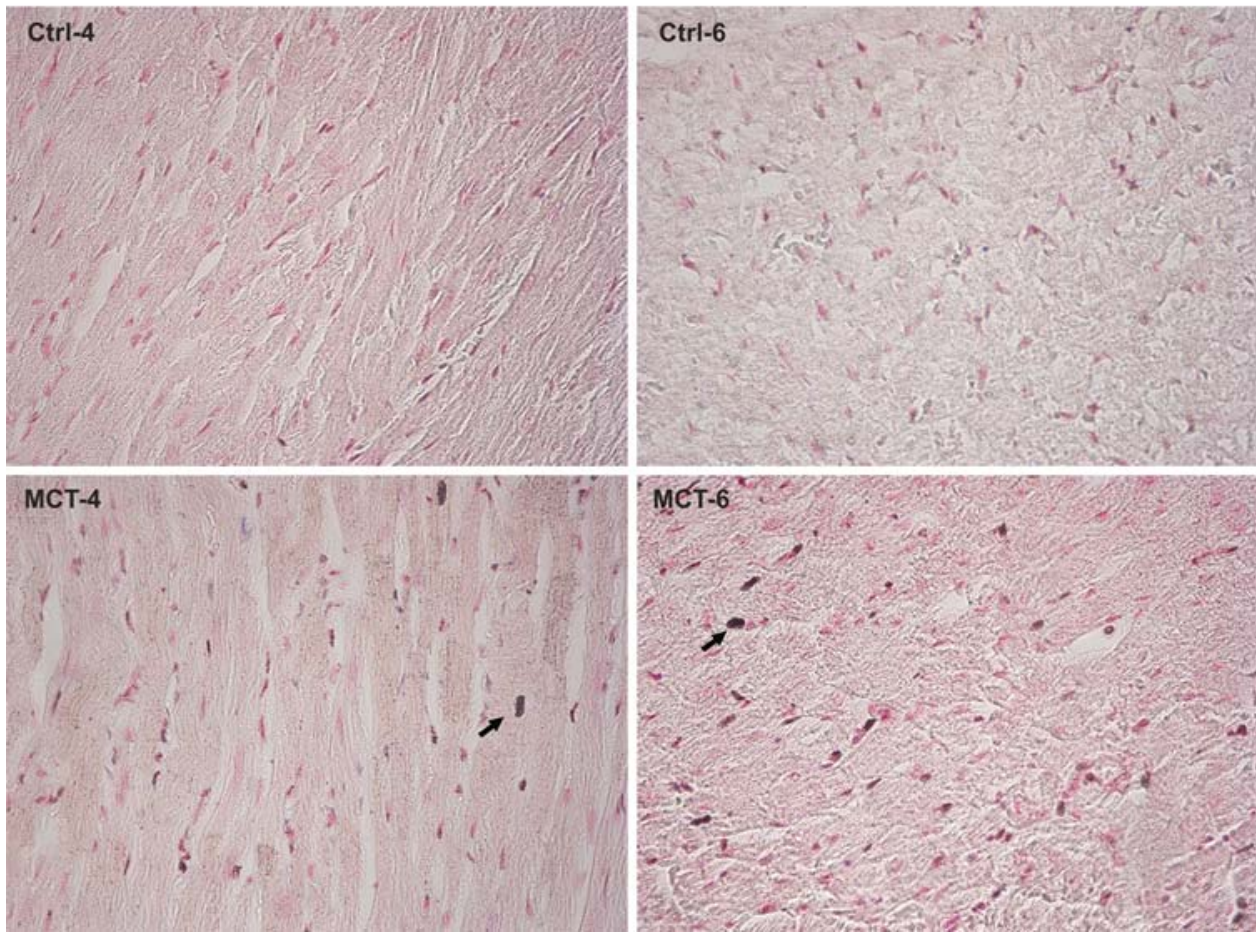

regulator proteins such as the $\mathrm{Ca}^{2+}$-ATPase of the sarcoendoplasmic reticulum and phospholamban were consistently reported to be unaltered in the non-overloaded LV [21, 42, 43]. To further explore the underlying mechanisms of LV myocardial dysfunction, we investigated neurohumoral activation, extracellular matrix composition and apoptosis.

Regarding local autocrine/paracrine related gene expression, MCT-4 rats showed increased ACE mRNA levels in the LV myocardium. However, only MCT-6 rats presented markedly increased expression of ET-1, and ACE was further upregulated compared with MCT-4. The activation of autocrine/paracrine systems, particularly for ET-1, was concomitant with the development of LV systolic and diastolic dysfunction in the baseline hemodynamic evaluation. It has been suggested that cardiac ET-1 may play a critical role in the functional deterioration of LV during the transition to congestive heart failure [18]. Indeed, chronic administration of ET-1, for 5 days, to engineered heart tissues, derived from cells of neonatal rat hearts, decreased the contractile response to calcium and isoprenaline and changed myosin heavy chain isoform and sarcoendoplasmic reticulum $\mathrm{Ca}^{2+}$-ATPase expression in a signaling pathway that involved protein kinase-C and the $\mathrm{Na}^{+} / \mathrm{H}^{+}$exchanger [51]. Lower heart rate, $\mathrm{LVP}_{\max }$ and $\mathrm{LV}$ dysfunction under the stress of anesthesia and surgical manipulation could well be partly the consequence of refractoriness to adrenergic stimulation mentioned above. Further supporting the possibility that ET-1 might have an important role modulating LV function in $\mathrm{PH}$ we have previously demonstrated that chronic ET-1 blockade prevents functional deterioration of the $\mathrm{LV}$ 
Fig. 8 Left ventricular (LV) mRNA levels of tenascin-C expressed as arbitrary units after normalization for GAPDH (a) was increased in MCT-6 group $(P<0.05: *$ vs. Ctrl-6; $\dagger$ vs. MCT-4). Collagen area fraction was increased in MCT-6 group $(P<0.05$ : * vs. Ctrl-6) (b). Representative examples of left ventricle myocardium stained with Masson's Trichrome of rats with monocrotaline-induced pulmonary hypertension (MCT) and controls (Ctrl) 4 and 6 weeks after injection, are presented in $\mathbf{c}$
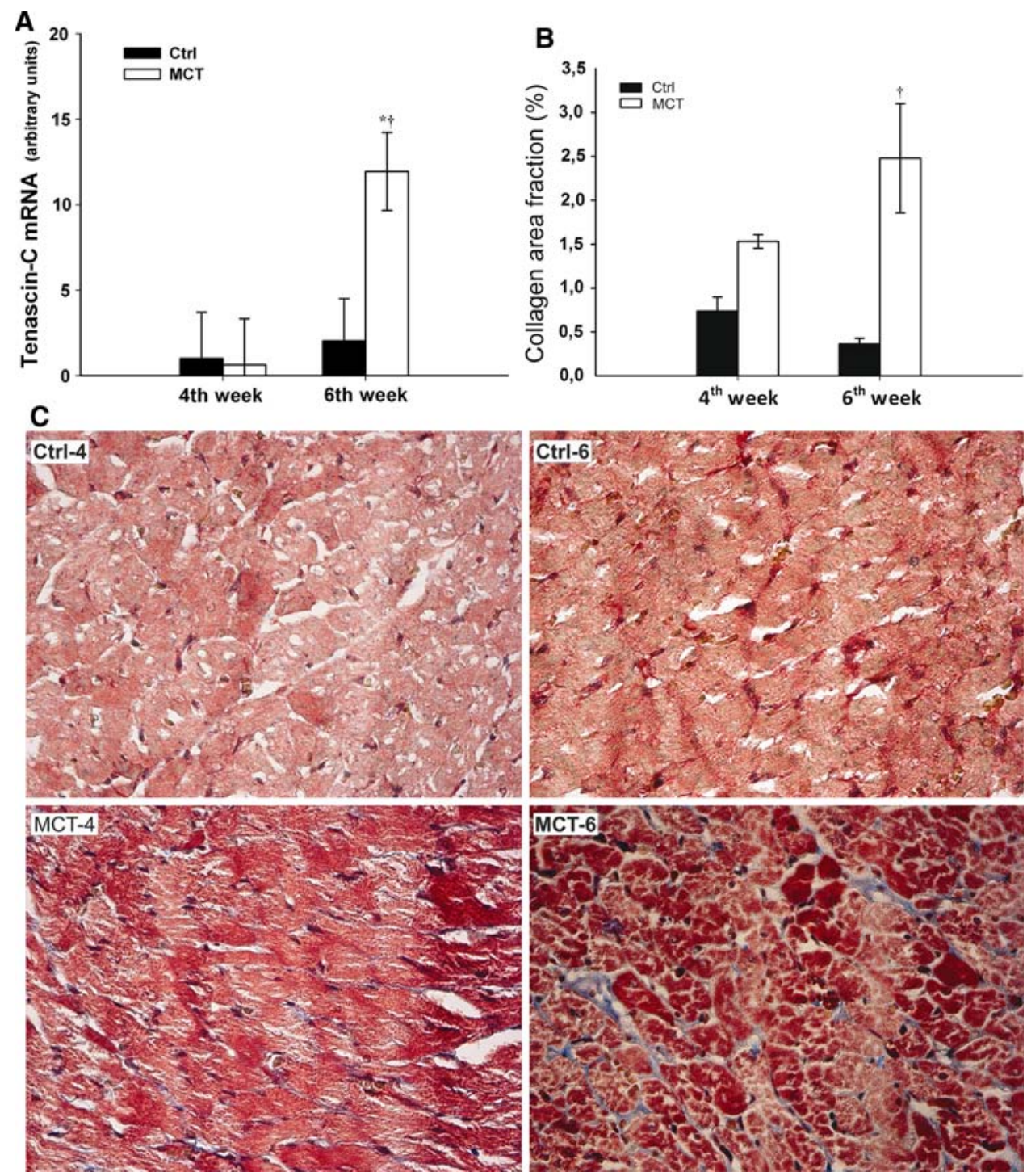

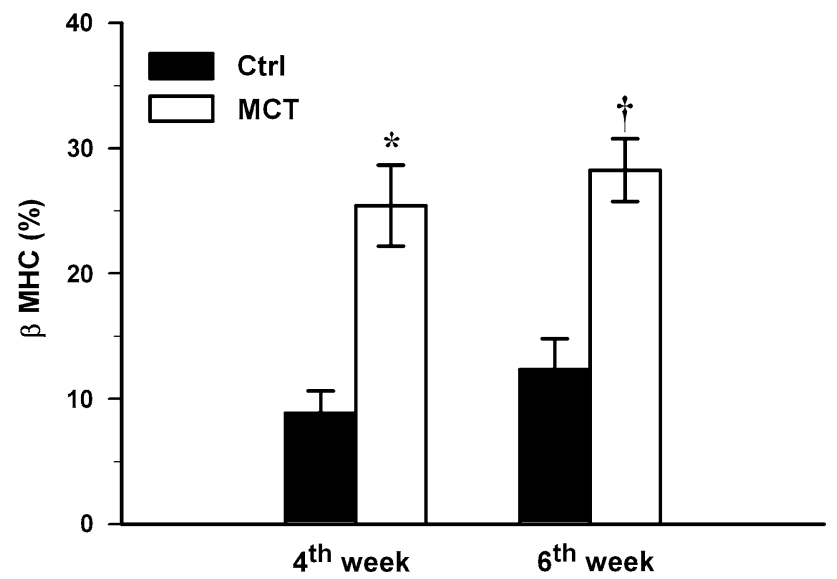

Fig. 9 Myosin heavy chain (MHC) isoform switch in monocrotalineinduced pulmonary hypertension, manifested as a significant increase of the $\beta$-MHC isoform that was similar in the MCT-4 and MCT-6 groups. $P<0.05$ : * vs. Ctrl-4; $\dagger$ vs. Ctrl-6 ( $n=6$ in each group) myocardium in $\mathrm{PH}$ rats, restoring normal contractile response to increasing frequencies of stimulation [33].

A possible molecular mechanism involved in the left ventricular dysfunction that accompanied severe $\mathrm{PH}$ is altered extracellular matrix composition and fibrillar collagen content $[6,8,38]$. Tenascin- $\mathrm{C}$ is synthesized by fibroblasts, highly expressed in embryonic tissues, and upregulated by both mechanical stress [2] and neuroendocrine mediators [34] in adult tissues under pathological conditions such as myocardial infarction [16]. Additionally, several pro-inflammatory cytokines, growth factors and neurohumoral peptides that stimulate tenascin-C synthesis [8, 34, 42] also participate in myocardial dysfunction induced by MCT [6, 33]. Tenascin-C is an important regulator of extracellular matrix and induces several metalloproteinases which activation is implicated in cardiac remodeling [44]. In the present study, we could demonstrate 
that there is a significant increase in myocardial interstitial collagen content and tenascin- $\mathrm{C}$ gene expression only 6 weeks after MCT injection, suggesting that changes in extracellular matrix composition may partly underlie the late deterioration of LV function in MCT-induced PH.

There are evidences suggesting that cell cycle and apoptosis pathways are co-activated in pressure-overload RV in MCT model [5]. In this sequence, we assessed the LV myocardial apoptosis, and found increased rates of apoptosis both in MCT-4 and MCT-6. Although there is some controversy about the role of apoptosis in chronic heart failure $[1,7,22]$, this results suggest that it might be another possible mechanism involved in early events of LV dysfunction.

In conclusion, selectively RV overloaded MCT-treated rats develop, on hemodynamic assessment, LV dysfunction that is accompanied by myosin heavy-chain isoform switch and local LV autocrine/paracrine system activation. Despite the changes in myocardial molecular phenotype, such as MHC isoform switch, increased apoptosis and ACE upregulation, in the LV, 4 weeks after MCT injection, diastolic dysfunction can only be detected in MCT-4 by increasing afterload. Two weeks later, however, the LV myocardium of MCT-6 overexpresses ET-1 has increased collagen content and augmented gene expression of tenascin-C and presents baseline systolic and diastolic dysfunction. The transition to LV dysfunction and congestive heart failure in $\mathrm{PH}$ is a complex process that involves the activation of several subcellular pathways. Afterload-dependent diastolic disturbances of the LV may herald, or contribute to, hemodynamic deterioration in $\mathrm{PH}$.

Acknowledgments Supported by Portuguese grants from FCT (POCI/SAU-FCF/60803/2004 and POCI/SAU-MMO/61547/2004) through Cardiovascular R\&D Unit (FCT No. 51/94). There are no conflicts of interest to disclose.

\section{References}

1. Anversa P, Kajstura J (1998) Myocyte cell death in the diseased heart. Circ Res 82:1231-1233

2. Chiquet M (1999) Regulation of extracellular matrix gene expression by mechanical stress. Matrix Biol 18:417-426

3. Correia-Pinto J, Henriques-Coelho T, Oliveira SM, Leite-Moreira AF (2003) Distinct load dependence of relaxation rate and diastolic function in Oryctolagus cuniculus and Ratus norvegicus. J Comp Physiol [B] 173:401-407

4. Correia Pinto J, Henriques-Coelho T, Roncon-Albuquerque R Jr, Leite-Moreira AF (2006) Differential right and left ventricular diastolic tolerance to acute afterload and NCX gene expression in Wistar rats. Physiol Res 55:513-526

5. Ecarnot-Laubriet A, Assem M, Poirson-Bichat F, Moisant M, Bernard C, Lecour S, Solary E, Rochette L, Teyssier JR (2002) Stage-dependent activation of cell cycle and apoptosis mechanisms in the right ventricle by pressure overload. Biochim Biophys Acta 1586:233-242
6. Fischer P, Hilfiker-Kleiner D (2007) Survival pathways in hypertrophy and heart failure: the gp130-STAT axis. Basic Res Cardiol 102:393-411

7. Freude B, Masters TN, Kostin S, Robicsek F, Schaper J (1998) Cardiomyocyte apoptosis in acute and chronic conditions. Basic Res Cardiol 93:85-89

8. Friehs I, Margossian RE, Moran AM, Cao-Danh H, Moses MA, del Nido PJ (2006) Vascular endothelial growth factor delays onset of failure in pressure-overload hypertrophy through matrix metalloproteinase activation and angiogenesis. Basic Res Cardiol 101:204-213

9. Gilbert JC, Glantz SA (1989) Determinants of left ventricular filling and of the diastolic pressure-volume relation. Circ Res 64:827-852

10. Gillebert TC, Leite-Moreira AF, De Hert SG (1997) Relaxationsystolic pressure relation. A load-independent assessment of left ventricular contractility. Circulation 95:745-752

11. Gomez A, Unruh H, Mink S (1994) Left ventricular systolic performance is depressed in chronic pulmonary emphysema in dogs. Am J Physiol 267:H232-H247

12. Gomez A, Unruh H, Mink SN (1993) Altered left ventricular chamber stiffness and isovolumic relaxation in dogs with chronic pulmonary hypertension caused by emphysema. Circulation $87: 247-260$

13. Grossman W, Barry WH (1980) Diastolic pressure-volume relations in the diseased heart. Fed Proc 39:148-155

14. Guerra MS, Roncon-Albuquerque R Jr, Lourenco AP, FalcaoPires I, Cibrao-Coutinho P, Leite-Moreira AF (2006) Remote myocardium gene expression after 30 and 120 min of ischaemia in the rat. Exp Physiol 91:473-480

15. Henriques-Coelho T, Correia-Pinto J, Roncon-Albuquerque R Jr, Baptista MJ, Lourenco AP, Oliveira SM, Brandao-Nogueira A, Teles A, Fortunato JM, Leite-Moreira AF (2004) Endogenous production of ghrelin and beneficial effects of its exogenous administration in monocrotaline-induced pulmonary hypertension. Am J Physiol Heart Circ Physiol 287:H2885-H2890

16. Imanaka-Yoshida K, Hiroe M, Nishikawa T, Ishiyama S, Shimojo T, Ohta Y, Sakakura T, Yoshida T (2001) Tenascin-C modulates adhesion of cardiomyocytes to extracellular matrix during tissue remodeling after myocardial infarction. Lab Invest 81:1015-1024

17. Ishikawa $S$, Honda $M$, Yamada $S$, Goto $Y$, Morioka $S$, Ishinaga Y, Murakami Y, Masumura S, Moriyama K (1992) Different biventricular remodelling of myosin and collagen in pulmonary hypertension. Clin Exp Pharmacol Physiol 19:723-732

18. Iwanaga $\mathrm{Y}$, Kihara $\mathrm{Y}$, Hasegawa $\mathrm{K}$, Inagaki $\mathrm{K}$, Yoneda $\mathrm{T}$, Kaburagi S, Araki M, Sasayama S (1998) Cardiac endothelin-1 plays a critical role in the functional deterioration of left ventricles during the transition from compensatory hypertrophy to congestive heart failure in salt-sensitive hypertensive rats. Circulation 98:2065-2073

19. Kawaguchi AT, Kawashima Y, Mizuta T, Ishibashi-Ueda H, Kanosue K, Shirakura R, Matsuda H (1992) Single lung transplantation in rats with fatal pulmonary hypertension. J Thorac Cardiovasc Surg 104:825-829

20. Kawaguchi M, Hay I, Fetics B, Kass DA (2003) Combined ventricular systolic and arterial stiffening in patients with heart failure and preserved ejection fraction: implications for systolic and diastolic reserve limitations. Circulation 107:714-720

21. Kobayashi H, Yoshimura Y, Suzuki H, Hosoda Y (1994) Regional difference of capillary-to-fiber ratio in the heart of monocrotaline-treated rats. Basic Res Cardiol 89:118-127

22. Kostin S, Pool L, Elsasser A, Hein S, Drexler HC, Arnon E, Hayakawa Y, Zimmermann R, Bauer E, Klovekorn WP, Schaper J (2003) Myocytes die by multiple mechanisms in failing human hearts. Circ Res 92:715-724 
23. Lamberts RR, Vaessen RJ, Westerhof N, Stienen GJ (2007) Right ventricular hypertrophy causes impairment of left ventricular diastolic function in the rat. Basic Res Cardiol 102:19-27

24. Larsen KO, Sjaastad I, Svindland A, Krobert KA, Skjonsberg $\mathrm{OH}$, Christensen G (2006) Alveolar hypoxia induces left ventricular diastolic dysfunction and reduces phosphorylation of phospholamban in mice. Am J Physiol Heart Circ Physiol 291:H507-H516

25. Leineweber K, Brandt K, Wludyka B, Beilfuss A, Ponicke K, Heinroth-Hoffmann I, Brodde OE (2002) Ventricular hypertrophy plus neurohumoral activation is necessary to alter the cardiac beta-adrenoceptor system in experimental heart failure. Circ Res 91:1056-1062

26. Leite-Moreira AF (2006) Current perspectives in diastolic dysfunction and diastolic heart failure. Heart 92:712-718

27. Leite-Moreira AF, Bras-Silva C, Pedrosa CA, Rocha-Sousa AA (2003) ET-1 increases distensibility of acutely loaded myocardium: a novel ETA and $\mathrm{Na}^{+} / \mathrm{H}^{+}$exchanger-mediated effect. Am J Physiol Heart Circ Physiol 284:H1332-H1339

28. Leite-Moreira AF, Castro-Chaves P, Pimentel-Nunes P, LimaCarneiro A, Guerra MS, Soares JB, Ferreira-Martins J (2006) Angiotensin II acutely decreases myocardial stiffness: a novel AT1, PKC and $\mathrm{Na}^{+} / \mathrm{H}^{+}$exchanger-mediated effect. Br J Pharmacol 147:690-697

29. Leite-Moreira AF, Correia-Pinto J (2001) Load as an acute determinant of end-diastolic pressure-volume relation. Am J Physiol Heart Circ Physiol 280:H51-H59

30. Leite-Moreira AF, Correia-Pinto J, Gillebert TC (1999) Afterload induced changes in myocardial relaxation: a mechanism for diastolic dysfunction. Cardiovasc Res 43:344-353

31. Leite-Moreira AF, Gillebert TC (1994) Nonuniform course of left ventricular pressure fall and its regulation by load and contractile state. Circulation 90:2481-2491

32. Louie EK, Rich S, Brundage BH (1986) Doppler echocardiographic assessment of impaired left ventricular filling in patients with right ventricular pressure overload due to primary pulmonary hypertension. J Am Coll Cardiol 8:1298-1306

33. Lourenco AP, Roncon-Albuquerque R Jr, Bras-Silva C, Faria B, Wieland J, Henriques-Coelho T, Correia-Pinto J, Leite-Moreira AF (2006) Myocardial dysfunction and neurohumoral activation without remodeling in left ventricle of monocrotaline-induced pulmonary hypertensive rats. Am J Physiol Heart Circ Physiol 291:H1587-H1594

34. Mackie EJ, Scott-Burden T, Hahn AW, Kern F, Bernhardt J, Regenass S, Weller A, Buhler FR (1992) Expression of tenascin by vascular smooth muscle cells. Alterations in hypertensive rats and stimulation by angiotensin II. Am J Pathol 141:377-388

35. Menzel T, Wagner S, Kramm T, Mohr-Kahaly S, Mayer E, Braeuninger S, Meyer J (2000) Pathophysiology of impaired right and left ventricular function in chronic embolic pulmonary hypertension: changes after pulmonary thromboendarterectomy. Chest 118:897-903

36. Miyauchi T, Yorikane R, Sakai S, Sakurai T, Okada M, Nishikibe M, Yano M, Yamaguchi I, Sugishita Y, Goto K (1993) Contribution of endogenous endothelin-1 to the progression of cardiopulmonary alterations in rats with monocrotaline-induced pulmonary hypertension. Circ Res 73:887-897

37. Paulus WJ, Shah AM (1999) NO and cardiac diastolic function. Cardiovasc Res 43:595-606

38. Perhonen M, Wang W, Han X, Ruskoaho H, Takala TE (1997) Right ventricular collagen type III and IV gene expression increases during early phases of endurance training in hypobaric hypoxic condition. Basic Res Cardiol 92:299-309

39. Pielsticker EJ, Martinez FJ, Rubenfire M (2001) Lung and heartlung transplant practice patterns in pulmonary hypertension centers. J Heart Lung Transplant 20:1297-1304

40. Roncon-Albuquerque $\mathrm{R} \mathrm{Jr}$, Vasconcelos $\mathrm{M}$, Lourenco AP, Brandao-Nogueira A, Teles A, Henriques-Coelho T, LeiteMoreira AF (2006) Acute changes of biventricular gene expression in volume and right ventricular pressure overload. Life Sci 78:2633-2642

41. Schena M, Clini E, Errera D, Quadri A (1996) Echo-Doppler evaluation of left ventricular impairment in chronic cor pulmonale. Chest 109:1446-1451

42. Schott P, Singer SS, Kogler H, Neddermeier D, Leineweber K, Brodde OE, Regitz-Zagrosek V, Schmidt B, Dihazi H, Hasenfuss G (2005) Pressure overload and neurohumoral activation differentially affect the myocardial proteome. Proteomics 5:1372-1381

43. Sharma S, Taegtmeyer H, Adrogue J, Razeghi P, Sen S, Ngumbela K, Essop MF (2004) Dynamic changes of gene expression in hypoxia-induced right ventricular hypertrophy. Am J Physiol Heart Circ Physiol 286:H1185-H1192

44. Spinale FG (2002) Matrix metalloproteinases: regulation and dysregulation in the failing heart. Circ Res 90:520-530

45. Sun XG, Hansen JE, Oudiz RJ, Wasserman K (2001) Exercise pathophysiology in patients with primary pulmonary hypertension. Circulation 104:429-435

46. Tuxworth WJ Jr, Shiraishi H, Moschella PC, Yamane K, McDermott PJ, Kuppuswamy D (2008) Translational activation of 5'-TOP mRNA in pressure overload myocardium. Basic Res Cardiol 103:41-53

47. Vescovo G, Harding SE, Jones SM, Dalla Libera L, Pessina AC, Poole-Wilson PA (1989) Comparison between isomyosin pattern and contractility of right ventricular myocytes isolated from rats with right cardiac hypertrophy. Basic Res Cardiol 84:536-543

48. Warner JG Jr, Metzger DC, Kitzman DW, Wesley DJ, Little WC (1999) Losartan improves exercise tolerance in patients with diastolic dysfunction and a hypertensive response to exercise. J Am Coll Cardiol 33:1567-1572

49. Weisfeldt ML, Frederiksen JW, Yin FC, Weiss JL (1978) Evidence of incomplete left ventricular relaxation in the dog: prediction from the time constant for isovolumic pressure fall. J Clin Invest 62:1296-1302

50. Xie GY, Lin CS, Preston HM, Taylor CG, Kearney K, Sapin PM, Smith MD (1998) Assessment of left ventricular diastolic function after single lung transplantation in patients with severe pulmonary hypertension. Chest $114: 477-481$

51. Zolk O, Munzel F, Eschenhagen T (2004) Effects of chronic endothelin-1 stimulation on cardiac myocyte contractile function. Am J Physiol Heart Circ Physiol 286:H1248-H1257 